\title{
ON THE EXTERIOR DIRICHLET PROBLEM FOR HESSIAN EQUATIONS
}

\author{
JIGUANG BAO, HAIGANG LI, AND YANYAN LI
}

\begin{abstract}
In this paper, we establish a theorem on the existence of the solutions of the exterior Dirichlet problem for Hessian equations with prescribed asymptotic behavior at infinity. This extends a result of Caffarelli and $\mathrm{Li}$ (2003) for the Monge-Ampère equation to Hessian equations.
\end{abstract}

\section{INTRODUCTION}

In this paper, we consider the solvability of the Dirichlet problem for Hessian equations

$$
\sigma_{k}\left(\lambda\left(D^{2} u\right)\right)=1
$$

on exterior domains $\mathbb{R}^{n} \backslash D$, where $D$ is a bounded open set in $\mathbb{R}^{n}, n \geq 3$, and $\lambda\left(D^{2} u\right)$ denotes the eigenvalues $\lambda_{1}, \cdots, \lambda_{n}$ of the Hessian matrix of $u$. Here

$$
\sigma_{k}(\lambda)=\sum_{1 \leq i_{1}<\cdots<i_{k} \leq n} \lambda_{i_{1}} \cdots \lambda_{i_{k}}
$$

is the $k$-th elementary symmetric function of $n$ variations, $k=1, \cdots, n$. Note that the case $k=1$ corresponds to Poisson's equation, which is a linear equation. There has been extensive literature on the exterior Dirichlet problem for linear elliptic equations of second order; see [19] and the references therein. For $2 \leq k \leq n$, the Hessian equation (1.1) is an important class of fully nonlinear elliptic equations. Especially, for $k=n$, we have the Monge-Ampère equation $\operatorname{det}\left(D^{2} u\right)=1$.

For the Monge-Ampère equation, a classical theorem of Jörgens ([17), Calabi ([5]), and Pogorelov ([20]) states that any classical convex solution of $\operatorname{det}\left(D^{2} u\right)=1$ in $\mathbb{R}^{n}$ must be a quadratic polynomial. A simpler and more analytic proof, along the lines of affine geometry, was later given by Cheng and Yau [6]. Caffarelli [1] extended the result for classical solutions to viscosity solutions. Another proof of this theorem was given by Jost and Xin in [18. Trudinger and Wang [24] proved that if $\Omega$ is an open convex subset of $\mathbb{R}^{n}$ and $u$ is a convex $C^{2}$ solution of $\operatorname{det}\left(D^{2} u\right)=1$ in $\Omega$ with $\lim _{x \rightarrow \partial \Omega} u(x)=\infty$, then $\Omega=\mathbb{R}^{n}$ and $u$ is quadratic.

Caffarelli and the third author [3] extended the Jörgens-Calabi-Pogorelov theorem to exterior domains. They proved that if $u$ is a convex viscosity solution of $\operatorname{det}\left(D^{2} u\right)=1$ outside a bounded subset of $\mathbb{R}^{n}, n \geq 3$, then there exist an $n \times n$ real symmetric positive definite matrix $A$, a vector $b \in \mathbb{R}^{n}$, and a constant $c \in \mathbb{R}$ such

Received by the editors April 12, 2012.

2010 Mathematics Subject Classification. Primary 35J60, 35J67.

The second author was the corresponding author. 
that

$$
\limsup _{|x| \rightarrow \infty}\left(|x|^{n-2}\left|u(x)-\left(\frac{1}{2} x^{T} A x+b \cdot x+c\right)\right|\right)<\infty .
$$

With this prescribed asymptotic behavior at infinity, an existence result for the exterior Dirichlet problem for the Monge-Ampère equation in $\mathbb{R}^{n}, n \geq 3$, was also established in 3 . In this paper, we will extend the existence theorem to the Dirichlet problem for Hessian equations (1.1) with $2 \leq k \leq n-1$ on exterior domains, with an appropriate asymptotic behavior at infinity. In dimension two, similar problems were studied by Ferrer, Martínez and Milán in [12,13] using the complex variable method. See also Delanoë [11.

We remark that for the case that $A=c^{*} I$, where

$$
c^{*}=\left(C_{n}^{k}\right)^{-1 / k}, \quad C_{n}^{k}=\frac{n !}{(n-k) ! k !},
$$

$I$ is the $n \times n$ identity matrix and $1 \leq k \leq n$, the exterior Dirichlet problem of Hessian equation (1.1) has been investigated in 9, 10. For interior domains, there have been many well-known results on the solvability of Hessian equations. For instance, Caffarelli, Nirenberg and Spruck 4 established the classical solvability of the Dirichlet problem, Trudinger [23] proved the existence and uniqueness of weak solutions, and Urbas 25] demonstrated the existence of viscosity solutions. Jian [16] studied the Hessian equations with infinite Dirichlet boundary value conditions.

For the reader's convenience, we recall the definition of viscosity solutions to Hessian equations (see [2,25] and the references therein). We say that a function $u \in C^{2}\left(\mathbb{R}^{n} \backslash \bar{D}\right)$ is admissible (or $k$-convex) if $\lambda\left(D^{2} u\right) \in \bar{\Gamma}_{k}$ in $\mathbb{R}^{n} \backslash \bar{D}$, where $\Gamma_{k}$ is the connected component of $\left\{\lambda \in \mathbb{R}^{n} \mid \sigma_{k}(\lambda)>0\right\}$ containing

$$
\Gamma^{+}=\left\{\lambda \in \mathbb{R}^{n} \mid \lambda_{i}>0, i=1, \cdots, n\right\} .
$$

It is well known that $\Gamma_{k}$ is a convex symmetric cone with vertex at the origin. Moreover,

$$
\Gamma_{k}=\left\{\lambda \in \mathbb{R}^{n} \mid \sigma_{j}(\lambda)>0, \text { for all } j=1, \cdots, k\right\} .
$$

See [4,22]. Clearly, $\Gamma_{k} \subseteq \Gamma_{j}$ for $k \geq j$, and $\Gamma_{1}$ is the half space $\left\{\lambda \in \mathbb{R}^{n} \mid \lambda_{1}+\cdots+\right.$ $\left.\lambda_{n}>0\right\}$, while $\Gamma_{n}=\Gamma^{+}$. We use the following definitions, which can be found in [21].

Let $\Omega \subset \mathbb{R}^{n}$; we use $\operatorname{USC}(\Omega)$ and $\operatorname{LSC}(\Omega)$ to denote respectively the set of upper and lower semicontinuous real valued functions on $\Omega$.

Definition 1.1. A function $u \in \operatorname{USC}\left(\mathbb{R}^{n} \backslash \bar{D}\right)$ is said to be a viscosity subsolution of equation (1.1) in $\mathbb{R}^{n} \backslash \bar{D}$ (or say that $u$ satisfies $\sigma_{k}\left(\lambda\left(D^{2} u\right)\right) \geq 1$ in $\mathbb{R}^{n} \backslash \bar{D}$ in the viscosity sense) if for any function $\psi \in C^{2}\left(\mathbb{R}^{n} \backslash \bar{D}\right)$ and point $\bar{x} \in \mathbb{R}^{n} \backslash \bar{D}$ satisfying

$$
\psi(\bar{x})=u(\bar{x}) \quad \text { and } \quad \psi \geq u \text { on } \mathbb{R}^{n} \backslash \bar{D},
$$

we have

$$
\sigma_{k}\left(\lambda\left(D^{2} \psi(\bar{x})\right)\right) \geq 1
$$

A function $u \in \operatorname{LSC}\left(\mathbb{R}^{n} \backslash \bar{D}\right)$ is said to be a viscosity supersolution of (1.1) in $\mathbb{R}^{n} \backslash \bar{D}$ (or say that $u$ satisfies $\sigma_{k}\left(\lambda\left(D^{2} u\right)\right) \leq 1$ in $\mathbb{R}^{n} \backslash \bar{D}$ in the viscosity sense) if for any $k$-convex function $\psi \in C^{2}\left(\mathbb{R}^{n} \backslash \bar{D}\right)$ and point $\bar{x} \in \mathbb{R}^{n} \backslash \bar{D}$ satisfying

$$
\psi(\bar{x})=u(\bar{x}) \quad \text { and } \quad \psi \leq u \text { on } \mathbb{R}^{n} \backslash \bar{D},
$$


we have

$$
\sigma_{k}\left(\lambda\left(D^{2} \psi(\bar{x})\right)\right) \leq 1
$$

A function $u \in C^{0}\left(\mathbb{R}^{n} \backslash \bar{D}\right)$ is said to be a viscosity solution of (1.1) if it is both a viscosity subsolution and supersolution of (1.1).

It is well known that a function $u \in C^{2}\left(\mathbb{R}^{n} \backslash \bar{D}\right)$ is a viscosity solution (respectively, subsolution, supersolution) of (1.1) if and only if it is a $k$-convex classical solution (respectively, subsolution, supersolution).

Definition 1.2. Let $\varphi \in C^{0}(\partial D)$. A function $u \in \operatorname{USC}\left(\mathbb{R}^{n} \backslash D\right)\left(u \in \operatorname{LSC}\left(\mathbb{R}^{n} \backslash D\right)\right)$ is said to be a viscosity subsolution (supersolution) of the Dirichlet problem

$$
\begin{cases}\sigma_{k}\left(\lambda\left(D^{2} u\right)\right)=1, & \text { in } \mathbb{R}^{n} \backslash \bar{D} \\ u=\varphi, & \text { on } \partial D\end{cases}
$$

if $u$ is a viscosity subsolution (supersolution) of (1.1) in $\mathbb{R}^{n} \backslash \bar{D}$ and $u \leq(\geq) \varphi$ on $\partial D$. A function $u \in C^{0}\left(\mathbb{R}^{n} \backslash D\right)$ is said to be a viscosity solution of (1.3) if it is both a subsolution and a supersolution.

Let

$\mathcal{A}_{k}=\left\{A \mid A\right.$ is a real $n \times n$ symmetric positive definite matrix, with $\left.\sigma_{k}(\lambda(A))=1\right\}$. Our main result is

Theorem 1.1. Let $D$ be a smooth, bounded, strictly convex open subset of $\mathbb{R}^{n}$, $n \geq 3$, and let $\varphi \in C^{2}(\partial D)$. Then for any given $b \in \mathbb{R}^{n}$ and any given $A \in \mathcal{A}_{k}$ with $2 \leq k \leq n$, there exists some constant $c_{*}$, depending only on $n, b, A, D$ and $\|\varphi\|_{C^{2}(\partial D)}$, such that for every $c>c_{*}$ there exists a unique viscosity solution $u \in$ $C^{0}\left(\mathbb{R}^{n} \backslash D\right)$ of $(1.3)$ and

$$
\limsup _{|x| \rightarrow \infty}\left(|x|^{\theta(n-2)}\left|u(x)-\left(\frac{1}{2} x^{T} A x+b \cdot x+c\right)\right|\right)<\infty,
$$

where $\theta \in\left[\frac{k-2}{n-2}, 1\right]$ is a constant depending only on $n, k$, and $A$.

Remark 1.1. For the two cases (i) $k=n$, the Monge-Ampère equations with any $A \in \mathcal{A}_{n}$, and (ii) $2 \leq k \leq n-1$, (1.4) with $A=c^{*} I \in \mathcal{A}_{k}$, Theorem 1.1 has been proved by Caffarelli-Li [3] and Dai-Bao [10, respectively, where $\theta=1$. Moreover, for the symmetric case $A=c^{*} I$, Wang-Bao [26] have proved that for $2 \leq k \leq n$, there exists a $\bar{c}(k, n)$ such that there is no classical radial solution of (1.3) and (1.4) if $c<\bar{c}(k, n)$.

Recall that any real symmetric matrix $A$ has an eigen-decomposition $A=O^{T} \Lambda O$ where $O$ is an orthogonal matrix and $\Lambda$ is a diagonal matrix. That is, $A$ may be regarded as a real diagonal matrix $\Lambda$ that has been re-expressed in some new coordinate system, and the eigenvalues $\lambda(A)=\lambda(\Lambda)$. Let

$$
y=O x \quad \text { and } \quad v(y)=u\left(O^{-1} y\right) .
$$

Then (1.3) and (1.4) become

$$
\begin{cases}\sigma_{k}\left(\lambda\left(D_{y}^{2} v\right)\right)=1, & \text { in } \mathbb{R}^{n} \backslash \overline{\widetilde{D}} \\ v=\varphi\left(O^{-1} y\right), & \text { on } \partial \widetilde{D}\end{cases}
$$


and

$$
\limsup _{|y| \rightarrow \infty}\left(\left|O^{-1} y\right|^{\theta(n-2)}\left|v(y)-\left(\frac{1}{2} y^{T} \Lambda y+b O^{-1} \cdot y+c\right)\right|\right)<\infty,
$$

where $\widetilde{D}$ is transformed from $D$ under $y=O x$. So, without loss of generality, we always assume that $A$ is diagonal in this paper.

If $A$ is diagonal and $A \in \mathcal{A}_{n}$, then $\sigma_{n}(\lambda(A))=1$, and we can find a diagonal matrix $Q$ with $\operatorname{det} Q=1$ such that $Q A Q=I \in \mathcal{A}_{n}$. Clearly, $\lambda(I)$ is not necessarily the same as $\lambda(A)$, but under the transformation $y=Q x$, we still have

$$
\operatorname{det}\left(D_{x}^{2} u\right)=\operatorname{det}\left(Q D_{y}^{2} u Q\right)=\operatorname{det}\left(D_{y}^{2} u\right) .
$$

Therefore, when the Monge-Ampère equation is considered, Caffarelli and Li 3$]$ can assume without loss of generality that $A=I$. However, when $2 \leq k \leq n-1$, if $A$ is diagonal and $A \in \mathcal{A}_{k}, \sigma_{k}(\lambda(A))=1$, although we can also find a diagonal matrix $Q$ such that $Q A Q=c^{*} I \in \mathcal{A}_{k}$, it is clear that $\lambda(A) \neq \lambda\left(c^{*} I\right)$ unless $A=c^{*} I$, and for the Hessian operator

$$
\sigma_{k}\left(\lambda\left(Q D_{y}^{2} u Q\right)\right) \neq \sigma_{k}(\lambda(Q)) \sigma_{k}\left(\lambda\left(D_{y}^{2} u\right)\right) \sigma_{k}(\lambda(Q)) .
$$

So, in order to prove Theorem 1.1 we are only allowed to assume that $A$ is diagonal, but we cannot further assume that $A=c^{*} I$.

Definition 1.3. For a diagonal matrix $A=\operatorname{diag}\left(a_{1}, a_{2}, \cdots, a_{n}\right)$, we call $u$ a generalized symmetric function with respect to $A$ if $u$ is a function of

$$
s=\frac{1}{2} x^{T} A x=\frac{1}{2} \sum_{i=1}^{n} a_{i} x_{i}^{2} .
$$

If $u$ is a generalized symmetric function with respect to $A$ and $u$ is a solution (respectively, subsolution, supersolution) of the Hessian equation (1.1), then we call $u$ a generalized symmetric solution (respectively, subsolution, supersolution) of (1.1).

In this paper we often abuse notation slightly by writing $u(x)=u\left(\frac{1}{2} x^{T} A x\right)$ for a generalized symmetric function with respect to $A$. Clearly, for diagonal matrix $A=\operatorname{diag}\left(a_{1}, a_{2}, \cdots, a_{n}\right) \in \mathcal{A}_{k}$ and real constants $\mu_{1}, \mu_{2}$, with $\mu_{1}^{k}=1$,

$$
\omega(s)=\mu_{1} s+\mu_{2}, \quad s=\frac{1}{2} \sum_{i=1}^{n} a_{i} x_{i}^{2}
$$

satisfies the Hessian equation (1.1) and $\omega^{\prime \prime}(s) \equiv 0$.

First, we will derive a formula of $\sigma_{k}(\lambda(M))$ for matrices $M$ of the form

$$
M=\left(p_{i} \delta_{i j}-\beta q_{i} q_{j}\right)_{n \times n},
$$

where $p=\left(p_{1}, p_{2}, \cdots, p_{n}\right), q=\left(q_{1}, q_{2}, \cdots, q_{n}\right)$ and $\beta \in \mathbb{R}$.

Proposition 1.2. If $M$ is an $n \times n$ matrix of the form (1.6) for $p=\left(p_{1}, p_{2}, \cdots, p_{n}\right)$, $q=\left(q_{1}, q_{2}, \cdots, q_{n}\right)$ and $\beta \in \mathbb{R}$, then we have

$$
\sigma_{k}(\lambda(M))=\sigma_{k}(p)-\beta \sum_{i=1}^{n} q_{i}^{2} \sigma_{k-1 ; i}(p),
$$

where $\sigma_{k-1 ; i}(p)=\left.\sigma_{k-1}(p)\right|_{p_{i}=0}$. 
For any $A=\operatorname{diag}\left(a_{1}, a_{2}, \cdots, a_{n}\right)$, suppose $\omega \in C^{2}\left(\mathbb{R}^{n}\right)$ is a generalized symmetric function with respect to $A$, that is,

$$
\omega(x)=\omega\left(\frac{1}{2} \sum_{i=1}^{n} a_{i} x_{i}^{2}\right) .
$$

Then

$$
\begin{gathered}
D_{i} \omega(x)=\omega^{\prime}(s) a_{i} x_{i}, \\
D_{i j} \omega(x)=\omega^{\prime}(s) a_{i} \delta_{i j}+\omega^{\prime \prime}(s)\left(a_{i} x_{i}\right)\left(a_{j} x_{j}\right) .
\end{gathered}
$$

We have the following lemma.

Lemma 1.3. For any $A=\operatorname{diag}\left(a_{1}, a_{2}, \cdots, a_{n}\right)$, if $\omega \in C^{2}\left(\mathbb{R}^{n}\right)$ is a generalized symmetric function with respect to $A$, then, with $a=\left(a_{1}, a_{2}, \cdots, a_{n}\right)$,

$$
\sigma_{k}\left(\lambda\left(D^{2} \omega\right)\right)=\sigma_{k}(a)\left(\omega^{\prime}\right)^{k}+\omega^{\prime \prime}\left(\omega^{\prime}\right)^{k-1} \sum_{i=1}^{n} \sigma_{k-1 ; i}(a)\left(a_{i} x_{i}\right)^{2} .
$$

If $A=c^{*} I, 2 \leq k \leq n$, then there exists a family of radially symmetric functions

$$
\bar{\omega}_{k}(s)=\int_{1}^{s}\left(1+\alpha t^{-\frac{n}{2}}\right)^{\frac{1}{k}} d t, \quad \alpha>0, s>0,
$$

satisfying

$$
\sigma_{k}\left(\lambda\left(D^{2} \omega\right)\right)=1, \quad \text { in } \mathbb{R}^{n} \backslash\{0\} .
$$

Such radially symmetric solutions play an important role in the solvability of the exterior Dirichlet problems studied by Caffarelli-Li [3] and by Dai-Bao [10]. However, for any given $A \in \mathcal{A}_{k}$ with $2 \leq k \leq n-1$, it is not enough to prove Theorem 1.1 by only using these radially symmetric functions. Due to the invariance of (1.1) for $k=n$, the Monge-Ampère equation, under affine transformations, $\bar{\omega}_{n}\left(\frac{1}{2} x^{T} A x\right)$ is a solution of (1.1) in $\mathbb{R}^{n} \backslash\{0\}$ for $A \in \mathcal{A}_{n}$. So the Monge-Ampère equation has generalized symmetric solutions with respect to $A$ for every $A \in \mathcal{A}_{n}$. A natural question is whether (1.1) with $2 \leq k \leq n-1$ has generalized symmetric solutions with respect to $A$ for every $A \in \mathcal{A}_{k}$ besides those of the form (1.5).

For this, we have

Proposition 1.4. For $A=\operatorname{diag}\left(a_{1}, a_{2}, \cdots, a_{n}\right) \in \mathcal{A}_{k}, 1 \leq k \leq n$, and $0<$ $\alpha<\beta<\infty$, if there exists an $\omega \in C^{2}(\alpha, \beta)$ with $\omega^{\prime \prime} \not \equiv 0$ in $(\alpha, \beta)$, such that $\omega(x)=\omega\left(\frac{1}{2} \sum_{i=1}^{n} a_{i} x_{i}^{2}\right)$ is a generalized symmetric solution of the Hessian equation (1.1) in $\left\{x \in \mathbb{R}^{n} \mid \alpha<\frac{1}{2} \sum_{i=1}^{n} a_{i} x_{i}^{2}<\beta\right\}$, then

$$
k=n \quad \text { or } \quad a_{1}=a_{2}=\cdots=a_{n}=c^{*},
$$

where $c^{*}=\left(C_{n}^{k}\right)^{-1 / k}, C_{n}^{k}=\frac{n !}{(n-k) ! k !}$, and vice versa.

This means that for $A=\operatorname{diag}\left(a_{1}, a_{2}, \cdots, a_{n}\right) \in \mathcal{A}_{k}, 2 \leq k \leq n-1, \omega\left(\frac{1}{2} x^{T} A x\right)$ is in general not a solution of (1.1).

To prove Theorem 1.1 for $2 \leq k \leq n-1$, it suffices to obtain enough subsolutions with appropriate properties. We construct such subsolutions which are generalized symmetric functions with respect to $A$. This is the main new ingredient in our proof of the theorem.

This paper is set out as follows. In the next section we construct a family of generalized symmetric smooth $k$-convex subsolutions of (1.1) in $\mathbb{R}^{n} \backslash\{0\}$. In Section 3. we prove Theorem 1.1 using Perron's method. 


\section{Generalized symmetric solutions and subsolutions}

In this section, we first derive formula (1.7) and (1.8), then prove Proposition 1.4. and finally construct a family of generalized symmetric smooth $k$-convex subsolutions of (1.1).

For $A=\operatorname{diag}\left(a_{1}, a_{2} \cdots, a_{n}\right)$, we denote $\lambda(A)=\left(a_{1}, a_{2} \cdots, a_{n}\right):=a$. If $A \in \mathcal{A}_{k}$, then we have $a_{i}>0(i=1,2, \cdots, n)$ and $\sigma_{k}(a)=1$. Here we introduce some notation. For any fixed $t$-tuple $\left\{i_{1}, \cdots, i_{t}\right\}, 1 \leq t \leq n-k$, we define

$$
\sigma_{k ; i_{1} \cdots i_{t}}(a)=\left.\sigma_{k}(a)\right|_{a_{i_{1}}=\cdots=a_{i_{t}}=0}
$$

that is, $\sigma_{k ; i_{1} \cdots i_{t}}$ is the $k$-th order elementary symmetric function of the $n-t$ variables $\left\{a_{i} \mid i \in\{1,2, \cdots, n\} \backslash\left\{i_{1}, i_{2}, \cdots, i_{t}\right\}\right\}$. The following properties of the functions $\sigma_{k}$ will be used in this paper:

$$
\sigma_{k}(a)=\sigma_{k ; i}(a)+a_{i} \sigma_{k-1 ; i}(a), i=1,2, \cdots, n,
$$

and

$$
\sum_{i=1}^{n} a_{i} \sigma_{k-1 ; i}(a)=k \sigma_{k}(a) .
$$

Now we prove Proposition 1.2 to derive a formula of $\sigma_{k}(\lambda(M))$ for matrices $M$ of the form (1.6).

Proof of Proposition 1.2. If $\beta=0$, (1.7) is obvious. If $\beta \neq 0$, we work with

$$
\widehat{M}=\frac{1}{\beta} M=\left(\hat{p}_{i} \delta_{i j}-q_{i} q_{j}\right), \quad \hat{p}=\frac{p}{\beta} .
$$

Therefore we only need to prove Proposition 1.2 for $\beta=1$, which we assume in the rest of the proof.

Denote

$$
D_{n}\left(\left\{p_{1}, p_{2}, \cdots, p_{n}\right\} ;\left\{q_{1}, q_{2}, \cdots, q_{n}\right\} ; \lambda\right):=\operatorname{det}(\lambda I-M) .
$$

By direct computations, we have

$$
\begin{aligned}
& D_{n}\left(\left\{p_{1}, p_{2}, \cdots, p_{n}\right\} ;\left\{q_{1}, q_{2}, \cdots, q_{n}\right\} ; \lambda\right) \\
& =\left|\begin{array}{ccccc}
\lambda-p_{1}+q_{1}^{2} & q_{1} q_{2} & \ldots & q_{1} q_{n-1} & q_{1} q_{n} \\
q_{2} q_{1} & \lambda-p_{2}+q_{2}^{2} & \ldots & q_{2} q_{n-1} & q_{2} q_{n} \\
\cdots & \cdots & \ldots & \cdots & \cdots \\
q_{n-1} q_{1} & q_{n-1} q_{2} & \cdots & \lambda-p_{n-1}+q_{n-1}^{2} & q_{n-1} q_{n} \\
q_{n} q_{1} & q_{n} q_{2} & \cdots & q_{n} q_{n-1} & \lambda-p_{n}+q_{n}^{2}
\end{array}\right| \\
& =\left|\begin{array}{ccccc}
\lambda-p_{1}+q_{1}^{2} & q_{1} q_{2} & \cdots & q_{1} q_{n-1} & 0 \\
q_{2} q_{1} & \lambda-p_{2}+q_{2}^{2} & \cdots & q_{2} q_{n-1} & 0 \\
\cdots & \cdots & \cdots & \cdots & \cdots \\
q_{n-1} q_{1} & q_{n-1} q_{2} & \cdots & \lambda-p_{n-1}+q_{n-1}^{2} & 0 \\
q_{n} q_{1} & q_{n} q_{2} & \cdots & q_{n} q_{n-1} & \lambda-p_{n}
\end{array}\right| \\
& +\left|\begin{array}{ccccc}
\lambda-p_{1}+q_{1}^{2} & q_{1} q_{2} & \ldots & q_{1} q_{n-1} & q_{1} q_{n} \\
q_{2} q_{1} & \lambda-p_{2}+q_{2}^{2} & \ldots & q_{2} q_{n-1} & q_{2} q_{n} \\
\ldots & \cdots & \cdots & \cdots & \cdots \\
q_{n-1} q_{1} & q_{n-1} q_{2} & \cdots & \lambda-p_{n-1}+q_{n-1}^{2} & q_{n-1} q_{n} \\
q_{n} q_{1} & q_{n} q_{2} & \cdots & q_{n} q_{n-1} & q_{n}^{2}
\end{array}\right|
\end{aligned}
$$




$$
\begin{aligned}
& =\left(\lambda-p_{n}\right) D_{n-1}\left(\left\{p_{1}, p_{2}, \cdots, p_{n-1}\right\} ;\left\{q_{1}, q_{2}, \cdots, q_{n-1}\right\} ; \lambda\right) \\
& +q_{n}\left|\begin{array}{ccccc}
\lambda-p_{1}+q_{1}^{2} & q_{1} q_{2} & \cdots & q_{1} q_{n-1} & q_{1} q_{n} \\
q_{2} q_{1} & \lambda-p_{2}+q_{2}^{2} & \cdots & q_{2} q_{n-1} & q_{2} q_{n} \\
\cdots & \cdots & \cdots & \cdots & \cdots \\
q_{n-1} q_{1} & q_{n-1} q_{2} & \cdots & \lambda-p_{n-1}+q_{n-1}^{2} & q_{n-1} q_{n} \\
q_{1} & q_{2} & \cdots & q_{n-1} & q_{n}
\end{array}\right| .
\end{aligned}
$$

For the second term, multiplying its last row by $-q_{i}(i \neq n)$ and adding to the $i$-th row, respectively, we obtain

$$
\begin{aligned}
& \left|\begin{array}{ccccc}
\lambda-p_{1}+q_{1}^{2} & q_{1} q_{2} & \ldots & q_{1} q_{n-1} & q_{1} q_{n} \\
q_{2} q_{1} & \lambda-p_{2}+q_{2}^{2} & \ldots & q_{2} q_{n-1} & q_{2} q_{n} \\
\cdots & \cdots & \cdots & \cdots & \cdots \\
q_{n-1} q_{1} & q_{n-1} q_{2} & \cdots & \lambda-p_{n-1}+q_{n-1}^{2} & q_{n-1} q_{n} \\
q_{1} & q_{2} & \cdots & q_{n-1} & q_{n}
\end{array}\right| \\
& =\left|\begin{array}{ccccc}
\lambda-p_{1} & 0 & \cdots & 0 & 0 \\
0 & \lambda-p_{2} & \cdots & 0 & 0 \\
\cdots & \cdots & \cdots & \cdots & \cdots \\
0 & 0 & \cdots & \lambda-p_{n-1} & 0 \\
q_{1} & q_{2} & \cdots & q_{n-1} & q_{n}
\end{array}\right| \\
& =q_{n}\left(\lambda-p_{1}\right)\left(\lambda-p_{2}\right) \cdots\left(\lambda-p_{n-1}\right) \text {. }
\end{aligned}
$$

Hence

$$
\begin{aligned}
& D_{n}\left(\left\{p_{1}, p_{2}, \cdots, p_{n}\right\} ;\left\{q_{1}, q_{2}, \cdots, q_{n}\right\} ; \lambda\right) \\
& =\left(\lambda-p_{n}\right) D_{n-1}\left(\left\{p_{1}, p_{2}, \cdots, p_{n-1}\right\} ;\left\{q_{1}, q_{2}, \cdots, q_{n-1}\right\} ; \lambda\right) \\
& \quad+q_{n}^{2}\left(\lambda-p_{1}\right)\left(\lambda-p_{2}\right) \cdots\left(\lambda-p_{n-1}\right) .
\end{aligned}
$$

We will deduce from (2.4), by induction, that for $n \geq 2$,

$$
D_{n}\left(\left\{p_{1}, p_{2}, \cdots, p_{n}\right\} ;\left\{q_{1}, q_{2}, \cdots, q_{n}\right\} ; \lambda\right)=\prod_{i=1}^{n}\left(\lambda-p_{i}\right)+\sum_{j=1}^{n}\left(q_{j}^{2} \prod_{i \neq j}\left(\lambda-p_{i}\right)\right) .
$$

For $n=2$,

$$
\begin{aligned}
D_{2}\left(\left\{p_{1}, p_{2}\right\} ;\left\{q_{1}, q_{2}\right\} ; \lambda\right) & =\left|\begin{array}{cc}
\lambda-p_{1}+q_{1}^{2} & q_{1} q_{2} \\
q_{1} q_{2} & \lambda-p_{2}+q_{2}^{2}
\end{array}\right| \\
& =\left(\lambda-p_{1}\right)\left(\lambda-p_{2}\right)+q_{1}^{2}\left(\lambda-p_{2}\right)+q_{2}^{2}\left(\lambda-p_{1}\right) .
\end{aligned}
$$


That is, (2.5) holds for $n=2$. We now assume (2.5) holds for $n-1 \geq 2$. Then by (2.4) and the induction hypothesis,

$$
\begin{aligned}
& D_{n}\left(\left\{p_{1}, p_{2}, \cdots, p_{n}\right\} ;\left\{q_{1}, q_{2}, \cdots, q_{n}\right\} ; \lambda\right) \\
& =\left(\lambda-p_{n}\right) D_{n-1}\left(\left\{p_{1}, p_{2}, \cdots, p_{n-1}\right\} ;\left\{q_{1}, q_{2}, \cdots, q_{n-1}\right\} ; \lambda\right) \\
& \quad+q_{n}^{2}\left(\lambda-p_{1}\right)\left(\lambda-p_{2}\right) \cdots\left(\lambda-p_{n-1}\right) \\
& =\left(\lambda-p_{n}\right)\left(\prod_{i=1}^{n-1}\left(\lambda-p_{i}\right)+\sum_{j=1}^{n-1}\left(q_{j}^{2} \prod_{i \neq j, i \leq n-1}\left(\lambda-p_{i}\right)\right)\right) \\
& \quad+q_{n}^{2}\left(\lambda-p_{1}\right)\left(\lambda-p_{2}\right) \cdots\left(\lambda-p_{n-1}\right) \\
& =\prod_{i=1}^{n}\left(\lambda-p_{i}\right)+\sum_{j=1}^{n}\left(q_{j}^{2} \prod_{i \neq j}\left(\lambda-p_{i}\right)\right) .
\end{aligned}
$$

We have proved that (2.5) holds for $n \geq 2$. Recall the Veite theorem that for any $n \times n$ matrix $U$,

$$
\operatorname{det}(\lambda I-U)=\sum_{i=0}^{n}(-1)^{i} \sigma_{i}(\lambda(U)) \lambda^{n-i} .
$$

In particular, if $U=\operatorname{diag}\left(p_{1}, p_{2} \cdots, p_{2}\right)$,

$$
\prod_{i=1}^{n}\left(\lambda-p_{i}\right)=\sum_{i=0}^{n}(-1)^{i} \sigma_{i}(p) \lambda^{n-i} ;
$$

here $p=\left(p_{1}, p_{2} \cdots, p_{n}\right)$. Using (2.3) and (2.7), (2.5) is written as

$$
\begin{aligned}
\operatorname{det}(\lambda I-M) & =\sum_{i=0}^{n}(-1)^{i} \sigma_{i}(p) \lambda^{n-i}+\sum_{j=1}^{n}\left(q_{j}^{2} \sum_{i=1}^{n}(-1)^{i-1} \sigma_{i-1 ; j}(p) \lambda^{n-i}\right) \\
& =\sum_{i=0}^{n}(-1)^{i}\left(\sigma_{i}(p)-\sum_{j=1}^{n} q_{j}^{2} \sigma_{i-1 ; j}(p)\right) \lambda^{n-i} .
\end{aligned}
$$

Here we used the standard conventions that $\sigma_{0}(p)=1$ and $\sigma_{-1}(p)=0$. Thus, (1.7) follows from (2.6). The proof of Proposition 1.2 is completed.

Proof of Lemma 1.3. For any $A=\operatorname{diag}\left(a_{1}, a_{2}, \cdots, a_{n}\right)$, if $\omega \in C^{2}\left(\mathbb{R}^{n}\right)$ is a generalized symmetric function with respect to $A$, that is,

$$
\omega(x)=\omega\left(\frac{1}{2} \sum_{i=1}^{n} a_{i} x_{i}^{2}\right),
$$

then

$$
\begin{gathered}
D_{i} \omega(x)=\omega^{\prime}(s) a_{i} x_{i}, \\
D_{i j} \omega(x)=\omega^{\prime}(s) a_{i} \delta_{i j}+\omega^{\prime \prime}(s)\left(a_{i} x_{i}\right)\left(a_{j} x_{j}\right) .
\end{gathered}
$$

Comparing (1.6) and (2.8), letting $\beta=-\omega^{\prime \prime}(s), p_{i}=\omega^{\prime}(s) a_{i}$ and $q_{i}=a_{i} x_{i}$, and substituting them into (1.7), we have (1.8). 
Symmetric solutions. For $A=c^{*} I$ and $2 \leq k \leq n$,

$$
\bar{\omega}_{k}(s)=\int_{1}^{s}\left(1+\alpha t^{-\frac{n}{2}}\right)^{\frac{1}{k}} d t, \quad \alpha>0, s>0,
$$

satisfies the ordinary differential equation

$$
\sigma_{k}\left(\lambda\left(D^{2} \omega\right)\right)=\left(\omega^{\prime}(s)\right)^{k}+2 s \frac{k}{n} \omega^{\prime \prime}(s)\left(\omega^{\prime}(s)\right)^{k-1}=1, \quad s>0 .
$$

Therefore, $\bar{\omega}_{k}\left(\frac{c^{*}}{2}|x|^{2}\right)$ is a solution of (1.1) in $\mathbb{R}^{n} \backslash\{0\}$. In order to prove Proposition 1.4. for every $a=\left(a_{1}, a_{2}, \cdots, a_{n}\right) \in \Gamma^{+}$, we denote

$$
A_{k}^{i}(a)=a_{i} \sigma_{k-1 ; i}(a), i=1,2, \cdots, n .
$$

From the property of $\sigma_{k},(2.2)$, we have

$$
\sum_{i=1}^{n} A_{k}^{i}(a)=k \sigma_{k}(a)
$$

Proof of Proposition 1.4. To better illustrate the idea of the proof, we start with $k=1$. For $s \in(\alpha, \beta), 1 \leq i \leq n$, let $x=\left(0, \cdots, 0, \sqrt{\frac{2 s}{a_{i}}}, 0, \cdots, 0\right)$. We have, using $A \in \mathcal{A}_{1}$,

$$
1=\Delta \omega(x)=\omega^{\prime}(s) \sum_{j=1}^{n} a_{j}+\omega^{\prime \prime}(s) \sum_{j=1}^{n} a_{j}^{2} x_{j}^{2}=\omega^{\prime}(s)+2 s \omega^{\prime \prime}(s) a_{i} .
$$

Since $\omega^{\prime \prime} \not \equiv 0$ in $(\alpha, \beta)$, there exists some $\bar{s} \in(\alpha, \beta)$ such that $\omega^{\prime \prime}(\bar{s}) \neq 0$. It follows that

$$
a_{i}=\frac{1-\omega^{\prime}(\bar{s})}{2 \bar{s} \omega^{\prime \prime}(\bar{s})}
$$

is independent of $i$. Since $A \in \mathcal{A}_{1}, 1=\sum_{i=1}^{n} a_{i}$. So $a_{1}=a_{2}=\cdots=a_{n}=\frac{1}{n}$. Proposition 1.4 for $k=1$ is established.

Now we consider the case $2 \leq k \leq n$. For $s \in(\alpha, \beta), 1 \leq i \leq n$, let $x=$ $\left(0, \cdots, 0, \sqrt{\frac{2 s}{a_{i}}}, 0, \cdots, 0\right)$. We have, using Lemma 1.3 ,

$$
\begin{aligned}
1 & =\sigma_{k}\left(\lambda\left(D^{2} \omega(x)\right)\right) \\
& =\sigma_{k}(a)\left(\omega^{\prime}(s)\right)^{k}+\omega^{\prime \prime}(s)\left(\omega^{\prime}(s)\right)^{k-1} \sigma_{k-1 ; j}(a)\left(a_{j} x_{j}\right)^{2} \\
& =\left(\omega^{\prime}(s)\right)^{k}+2 s \omega^{\prime \prime}(s)\left(\omega^{\prime}(s)\right)^{k-1} \sigma_{k-1 ; i}(a) a_{i} .
\end{aligned}
$$

It is clear from the above that $\omega^{\prime}(s) \neq 0, \forall s \in(\alpha, \beta)$. Since $\omega^{\prime \prime} \not \equiv 0$ in $(\alpha, \beta)$, there exists some $\bar{s} \in(\alpha, \beta)$ such that $\omega^{\prime \prime}(\bar{s}) \neq 0$. It follows that

$$
A_{k}^{i}(a)=\sigma_{k-1 ; i}(a) a_{i}=\frac{1-\left(\omega^{\prime}(\bar{s})\right)^{k}}{2 \bar{s} \omega^{\prime \prime}(\bar{s})\left(\omega^{\prime}(\bar{s})\right)^{k-1}}
$$

is independent of $i$. For $2 \leq k \leq n-1$, for any $i_{1}, i_{2} \in\{1,2, \cdots, n\}$, by (2.11) and (2.1) we have

$$
\begin{aligned}
0 & =A_{k}^{i_{1}}(a)-A_{k}^{i_{2}}(a) \\
& =a_{i_{1}} \sigma_{k-1 ; i_{1}}(a)-a_{i_{2}} \sigma_{k-1 ; i_{2}}(a) \\
& =a_{i_{1}}\left(a_{i_{2}} \sigma_{k-2 ; i_{1} i_{2}}(a)+\sigma_{k-1 ; i_{1} i_{2}}(a)\right)-a_{i_{2}}\left(a_{i_{1}} \sigma_{k-2 ; i_{1} i_{2}}(a)+\sigma_{k-1 ; i_{1} i_{2}}(a)\right) \\
& =\left(a_{i_{1}}-a_{i_{2}}\right) \sigma_{k-1 ; i_{1} i_{2}}(a) .
\end{aligned}
$$


Since $a_{i}>0, i=1,2, \cdots, n$, it follows that $\sigma_{k-1 ; i_{1} i_{2}}(a) \neq 0$. By the arbitrariness of $i_{1}, i_{2}$, we have $a_{1}=a_{2}=\cdots=a_{n}$. Using $\sigma_{k}(a)=1$, we have

$$
a_{1}=a_{2}=\cdots=a_{n}=\left(C_{n}^{k}\right)^{-1 / k} .
$$

Proposition 1.4 is proved.

Generalized symmetric subsolutions. From Proposition 1.4, we see that there are no generalized symmetric solutions of (1.1) with $\omega^{\prime \prime}(s) \not \equiv 0$ in the remaining cases. We will construct a family of generalized symmetric smooth functions satisfying

$$
\omega^{\prime}(s)>0, \quad \omega^{\prime \prime}(s) \leq 0
$$

and

$$
\sigma_{k}\left(\lambda\left(D^{2} \omega\right)\right) \geq 1 \quad \text { and } \quad \sigma_{m}\left(\lambda\left(D^{2} \omega\right)\right) \geq 0,1 \leq m \leq k-1 .
$$

For $A=\operatorname{diag}\left(a_{1}, a_{2}, \cdots, a_{n}\right) \in \mathcal{A}_{k}$, denote $a=\left(a_{1}, a_{2}, \cdots, a_{n}\right)$, and consider

$$
h_{k}(a):=\max _{1 \leq i \leq n} A_{k}^{i}(a) .
$$

Since $A_{n}^{i}(a)=a_{i} \sigma_{n-1 ; i}(a)=\sigma_{n}(a)$ for every $i$, we have $h_{n}(a)=1$. By (2.11), (2.1) and (2.12), we have, for $1 \leq k \leq n-1$,

$$
A_{k}^{i}(a)=a_{i} \sigma_{k-1 ; i}(a)<\sigma_{k}(a)=1, \quad \forall i,
$$

and

$$
n h_{k}(a) \geq \sum_{i=1}^{n} A_{k}^{i}(a)=k \sigma_{k}(a)=k .
$$

We see from the above that

$$
\frac{k}{n} \leq h_{k}(a)<1
$$

where " $="$ holds if and only if $A_{k}^{i}(a)$ is independent of $i$, i.e., in view of (2.13), $a_{1}=a_{2}=\cdots=a_{n}=c^{*}$. For $n \geq 3$ and $2 \leq k \leq n$, in view of (2.15) and $h_{n}(a)=1$, we have

$$
\frac{k}{2 h_{k}(a)}>1
$$

By a simple computation, the ordinary differential equation

$$
\left\{\begin{array}{l}
\left(\omega^{\prime}(s)\right)^{k}+2 h_{k}(a) s \omega^{\prime \prime}(s)\left(\omega^{\prime}(s)\right)^{k-1}=1, \quad s>0, \\
\omega^{\prime}(s)>0, \quad \omega^{\prime \prime}(s) \leq 0
\end{array}\right.
$$

has a family of solutions

$$
\omega_{\alpha}(s)=\beta+\int_{\bar{s}}^{s}\left(1+\alpha t^{-\frac{k}{2 h_{k}(a)}}\right)^{\frac{1}{k}} d t, \quad \alpha>0, s>0,
$$

where $\beta \in \mathbb{R}$ and $\bar{s}>0$. It follows from (2.16) that

$$
\begin{aligned}
\omega_{\alpha}(s) & =\beta+s-\bar{s}+\int_{\bar{s}}^{s}\left(\left(1+\alpha t^{-\frac{k}{2 h_{k}(a)}}\right)^{\frac{1}{k}}-1\right) d t \\
& =s+\mu(\alpha)+O\left(s^{\frac{(2-n) \theta}{2}}\right), \quad \text { as } \quad s \rightarrow \infty,
\end{aligned}
$$

where

$$
\mu(\alpha)=\beta-\bar{s}+\int_{\bar{s}}^{\infty}\left(\left(1+\alpha t^{-\frac{k}{2 h_{k}(a)}}\right)^{\frac{1}{k}}-1\right) d t<\infty
$$


and

$$
\theta=\frac{1}{n-2}\left(\frac{k}{h_{k}(a)}-2\right) .
$$

We see from (2.15) that $\theta \in\left(\frac{k-2}{n-2}, 1\right]$ if $2 \leq k \leq n-1$ and $\theta=1$ if $k=n$.

Proposition 2.1. For $n \geq 3$ and $2 \leq k \leq n, A \in \mathcal{A}_{k}$, let $\omega_{\alpha}(x)=\omega_{\alpha}\left(\frac{1}{2} x^{T} A x\right)$ be given in (2.18). Then $\omega_{\alpha}$ is a smooth $k$-convex subsolution of (1.1) in $\mathbb{R}^{n} \backslash\{0\}$ satisfying

$$
\omega_{\alpha}(x)=\frac{1}{2} x^{T} A x+\mu(\alpha)+O\left(|x|^{\theta(2-n)}\right), \quad \text { as } \quad x \rightarrow \infty .
$$

Proof. Obviously, (2.20) follows from (2.19). By computation,

$$
\begin{gathered}
\omega_{\alpha}^{\prime}(s)=\left(1+\alpha s^{-\frac{k}{2 h_{k}(a)}}\right)^{\frac{1}{k}}>1, \\
\omega_{\alpha}^{\prime \prime}(s)=-\frac{1}{2 h_{k}(a) s} \cdot \frac{\alpha}{s^{\frac{k}{2 h_{k}(a)}}+\alpha} \cdot \omega_{\alpha}^{\prime}(s)<0 .
\end{gathered}
$$

It is clear from Lemma 1.3, (2.14) and (2.17) that

$$
\sigma_{k}\left(\lambda\left(D^{2} u\right)\right) \geq \sigma_{k}(a)\left(\omega_{\alpha}^{\prime}\right)^{k}+h_{k}(a) \omega_{\alpha}^{\prime \prime}\left(\omega_{\alpha}^{\prime}\right)^{k-1} 2 s=1, \quad \text { in } \mathbb{R}^{n} \backslash\{0\} .
$$

By Lemma 1.3. (2.21) and (2.14), we have, for any $1 \leq m \leq k-1$,

$$
\begin{aligned}
\sigma_{m}\left(\lambda\left(D^{2} u\right)\right) & =\sigma_{m}(a)\left(\omega_{\alpha}^{\prime}\right)^{m}+\omega_{\alpha}^{\prime \prime}\left(\omega_{\alpha}^{\prime}\right)^{m-1} \sum_{i=1}^{n} \sigma_{m-1 ; i}(a)\left(a_{i} x_{i}\right)^{2} \\
& =\left(\omega_{\alpha}^{\prime}\right)^{m}\left(\sigma_{m}(a)-\frac{1}{2 s h_{k}(a)} \cdot \frac{\alpha}{s^{\frac{k}{2 h_{k}(a)}}+\alpha} \sum_{i=1}^{n} \sigma_{m-1 ; i}(a)\left(a_{i} x_{i}\right)^{2}\right) \\
& \geq\left(\omega_{\alpha}^{\prime}\right)^{m}\left(\sigma_{m}(a)-\frac{1}{2 s} \cdot \frac{\alpha}{s^{\frac{k}{2 h_{k}(a)}}+\alpha} \sum_{i=1}^{n} \frac{\sigma_{m-1 ; i}(a)\left(a_{i} x_{i}\right)^{2}}{a_{i} \sigma_{k-1 ; i}(a)}\right) .
\end{aligned}
$$

In order to show $\sigma_{m}\left(\lambda\left(D^{2} u\right)\right) \geq 0$, it suffices to prove, for each $1 \leq i \leq n$,

$$
\sigma_{m}(a) \sigma_{k-1 ; i}(a) \geq \sigma_{m-1 ; i}(a) .
$$

Note that the Newtonian inequalities may be expressed as

$$
\frac{\sigma_{k+1}(a)}{C_{n}^{k+1}} \cdot \frac{\sigma_{k-1}(a)}{C_{n}^{k-1}} \leq\left(\frac{\sigma_{k}(a)}{C_{n}^{k}}\right)^{2},
$$

for $1 \leq k \leq n-1$. Since

$$
\frac{C_{n}^{k-1} C_{n}^{k+1}}{C_{n}^{k} C_{n}^{k}}=\frac{(n-k) k}{(n-k+1)(k+1)}<1,
$$

it follows that

$$
\frac{\sigma_{k+1}(a)}{\sigma_{k}(a)} \leq \frac{\sigma_{k}(a)}{\sigma_{k-1}(a)}
$$

which shows that the Hessian quotient $\frac{\sigma_{k+1}(a)}{\sigma_{k}(a)}$ is decreasing with respect to $k$. So we have for any $m \leq k$, and each $1 \leq i \leq n$,

$$
\sigma_{m ; i}(a) \sigma_{k-1 ; i}(a) \geq \sigma_{m-1 ; i}(a) \sigma_{k ; i}(a) .
$$


Then by the property (2.1), it follows that

$$
\begin{aligned}
\sigma_{m}(a) \sigma_{k-1 ; i}(a) & =\left(\sigma_{m ; i}(a)+a_{i} \sigma_{m-1 ; i}(a)\right) \sigma_{k-1 ; i}(a) \\
& \geq \sigma_{m-1 ; i}(a) \cdot \sigma_{k ; i}(a)+\sigma_{m-1 ; i}(a) \cdot a_{i} \sigma_{k-1 ; i}(a) \\
& =\sigma_{m-1 ; i}(a) \sigma_{k}(a) \\
& =\sigma_{m-1 ; i}(a)
\end{aligned}
$$

i.e. (2.22) is proved. Hence $\omega_{\alpha}$ is a smooth $k$-convex subsolution of (1.1) in $\mathbb{R}^{n} \backslash\{0\}$.

\section{Proof of Theorem 1.1}

The following lemma holds for any invertible and symmetric matrix $A$, and where $A$ is not necessarily diagonal or in $\mathcal{A}_{k}, 2 \leq k \leq n$.

Lemma 3.1. Let $\varphi \in C^{2}(\partial D)$. There exists some constant $C$, depending only on $n,\|\varphi\|_{C^{2}(\partial D)}$, the upper bound of $A$, the diameter and the convexity of $D$, and the $C^{2}$ norm of $\partial D$, such that, for every $\xi \in \partial D$, there exists $\bar{x}(\xi) \in \mathbb{R}^{n}$ satisfying

$$
|\bar{x}(\xi)| \leq C \quad \text { and } \quad w_{\xi}<\varphi \text { on } \bar{D} \backslash\{\xi\},
$$

where

$$
w_{\xi}(x):=\varphi(\xi)+\frac{1}{2}\left((x-\bar{x}(\xi))^{T} A(x-\bar{x}(\xi))-(\xi-\bar{x}(\xi))^{T} A(\xi-\bar{x}(\xi))\right), \quad x \in \mathbb{R}^{n} .
$$

Proof. Let $\xi \in \partial D$. By a translation and a rotation, we may assume without loss of generality that $\xi=0$ and $\partial D$ is locally represented by the graph of

$$
x_{n}=\rho\left(x^{\prime}\right)=O\left(\left|x^{\prime}\right|^{2}\right),
$$

and $\varphi$ locally has the expansion

$$
\begin{aligned}
\varphi\left(x^{\prime}, \rho\left(x^{\prime}\right)\right) & =\varphi(0)+\varphi_{x_{1}}(0) x_{1}+\cdots+\varphi_{x_{n}}(0) x_{n}+O\left(|x|^{2}\right) \\
& =\varphi(0)+\varphi_{x_{1}}(0) x_{1}+\cdots+\varphi_{x_{n-1}}(0) x_{n-1}+O\left(\left|x^{\prime}\right|^{2}\right),
\end{aligned}
$$

where $x^{\prime}=\left(x_{1}, \cdots, x_{n-1}\right)$.

Since $A$ is invertible, we can find $\bar{x}=\bar{x}(t) \in \mathbb{R}^{n}$ such that, for appropriate $t$ to fit our need later,

$$
A \bar{x}(t)=\left(-\varphi_{x_{1}}(0), \cdots,-\varphi_{x_{n-1}}(0), t\right)^{T}
$$

Let

$$
w(x)=\varphi(0)+\frac{1}{2}\left((x-\bar{x})^{T} A(x-\bar{x})-\bar{x}^{T} A \bar{x}\right), \quad x \in \mathbb{R}^{n} .
$$

Then

$$
w(x)=\varphi(0)+\frac{1}{2} x^{T} A x-x^{T} A \bar{x}=\varphi(0)+\frac{1}{2} x^{T} A x+\sum_{\alpha=1}^{n-1} \varphi_{x_{\alpha}}(0) x_{\alpha}-t x_{n} .
$$

It follows that

$$
\begin{aligned}
(w-\varphi)\left(x^{\prime}, \rho\left(x^{\prime}\right)\right) & =\frac{1}{2} x^{T} A x-t \rho\left(x^{\prime}\right)+O\left(\left|x^{\prime}\right|^{2}\right) \\
& \leq C\left(\left|x^{\prime}\right|^{2}+\rho\left(x^{\prime}\right)^{2}\right)-t \rho\left(x^{\prime}\right)
\end{aligned}
$$


where $C$ depends only on the upper bound of $A,\|\varphi\|_{C^{2}(\partial D)}$, and the $C^{2}$ norm of $\partial D$. By the strict convexity of $\partial D$, there exists some constant $\delta>0$ depending only on $D$ such that

$$
\rho\left(x^{\prime}\right) \geq \delta\left|x^{\prime}\right|^{2}, \quad \forall\left|x^{\prime}\right|<\delta .
$$

Clearly, for large $t$, we have

$$
(w-\varphi)\left(x^{\prime}, \rho\left(x^{\prime}\right)\right)<0, \quad \forall 0<\left|x^{\prime}\right|<\delta .
$$

The largeness of $t$ depends only on $\delta, A,\|\varphi\|_{C^{2}(\partial D)}$, and the $C^{2}$ norm of $\partial D$.

On the other hand, by the strict convexity of $\partial D$ and (3.2),

$$
x_{n} \geq \delta^{3}, \quad \forall x \in \partial D \backslash\left\{\left(x^{\prime}, \rho\left(x^{\prime}\right)\right)|| x^{\prime} \mid<\delta\right\} .
$$

It follows from (3.1) that

$$
w(x) \leq C-\delta^{3} t, \forall x \in \partial D \backslash\left\{\left(x^{\prime}, \rho\left(x^{\prime}\right)\right)|| x^{\prime} \mid<\delta\right\},
$$

where $C$ depends only on $A, \operatorname{diam}(D)$, and $\|\varphi\|_{C^{2}(\partial D)}$. By making $t$ large (still under control), we have

$$
w(x)-\varphi(x)<0, \forall x \in \partial D \backslash\left\{\left(x^{\prime}, \rho\left(x^{\prime}\right)\right)|| x^{\prime} \mid<\delta\right\} .
$$

Lemma 3.1 is established.

By an orthogonal transformation and by subtracting a linear function from $u$, we only need to prove Theorem 1.1 for the case that $A=\operatorname{diag}\left(a_{1}, a_{2}, \cdots, a_{n}\right)$, where $a_{i}>0(1 \leq i \leq n), b=0$.

Proof of Theorem 1.1. Without loss of generality, we assume that $0 \in D$. For $s>0$, let

$$
E(s):=\left\{x \in \mathbb{R}^{n} \mid \frac{1}{2} x^{T} A x<s\right\} .
$$

Fix $\bar{s}>0$ such that $\bar{D} \subset E(\bar{s})$. For $\alpha>0, \beta \in \mathbb{R}$, set

$$
\omega_{\alpha}(x)=\beta+\int_{\bar{s}}^{\frac{1}{2} x^{T} A x}\left(1+\alpha t^{-\frac{k}{2 h_{k}(a)}}\right)^{\frac{1}{k}} d t,
$$

as in (2.18). We have by Proposition 2.1 that $\omega_{\alpha}$ is a smooth $k$-convex subsolution of (1.1) in $\mathbb{R}^{n} \backslash\{0\}$ and that

$$
\omega_{\alpha}(x)=\frac{1}{2} x^{T} A x+\mu(\alpha)+O\left(|x|^{\theta(2-n)}\right), \quad \text { as } \quad x \rightarrow \infty .
$$

Here

$$
\mu(\alpha)=\beta-\bar{s}+\int_{\bar{s}}^{\infty}\left(\left(1+\alpha t^{-\frac{k}{2 h_{k}(a)}}\right)^{\frac{1}{k}}-1\right) d t, \quad \theta \in\left[\frac{k-2}{n-2}, 1\right] .
$$

Clearly, $\mu(\alpha)$ is strictly increasing in $\alpha$, and

$$
\lim _{\alpha \rightarrow \infty} \mu(\alpha)=\infty \text {. }
$$

On the other hand,

$$
\omega_{\alpha} \leq \beta, \quad \text { in } E(\bar{s}) \backslash \bar{D}, \forall \alpha>0 \text {. }
$$

Let

$$
\begin{aligned}
& \beta:=\min \left\{w_{\xi}(x) \mid \xi \in \partial D, x \in \overline{E(\bar{s})} \backslash D\right\}, \\
& \widehat{b}:=\max \left\{w_{\xi}(x) \mid \xi \in \partial D, x \in \overline{E(\bar{s})} \backslash D\right\},
\end{aligned}
$$


where $w_{\xi}(x)$ is given by Lemma 3.1. We will fix the value of $c_{*}$ in the proof. First we require that $c_{*}$ satisfies $c_{*}>\widehat{b}$. It follows that

$$
\mu(0)=\beta-\bar{s}<\beta \leq \widehat{b}<c_{*} .
$$

Thus, in view of (3.3), for every $c>c_{*}$ there exists a unique $\alpha(c)$ such that

$$
\mu(\alpha(c))=c .
$$

So $\omega_{\alpha(c)}$ satisfies

$$
\omega_{\alpha(c)}(x)=\frac{1}{2} x^{T} A x+c+O\left(|x|^{\theta(2-n)}\right), \quad \text { as } \quad x \rightarrow \infty .
$$

Set

$$
\underline{w}(x)=\max \left\{w_{\xi}(x) \mid \xi \in \partial D\right\} .
$$

It is clear by Lemma 3.1 that $\underline{w}$ is a locally Lipschitz function in $\mathbb{R}^{n} \backslash D$ and that $\underline{w}=\varphi$ on $\partial D$. Since $w_{\xi}$ is a smooth convex solution of (1.1), $\underline{w}$ is a viscosity subsolution of (1.1) in $\mathbb{R}^{n} \backslash \bar{D}$. We fix a number $\hat{s}>\bar{s}$ and then choose another number $\widehat{\alpha}>0$ such that

$$
\min _{\partial E(\hat{s})} \omega_{\widehat{\alpha}}>\max _{\partial E(\hat{s})} \underline{w} .
$$

We require that $c_{*}$ also satisfies $c_{*} \geq \mu(\widehat{\alpha})$. We now fix the value of $c_{*}$.

For $c \geq c_{*}$, we have $\alpha(c)=\mu^{-1}(c) \geq \mu^{-1}\left(c_{*}\right) \geq \widehat{\alpha}$, and therefore

$$
\omega_{\alpha(c)} \geq \omega_{\widehat{\alpha}}>\underline{w}, \quad \text { on } \partial E(\hat{s}) .
$$

By (3.4), we have

$$
\omega_{\alpha(c)} \leq \beta \leq \underline{w}, \quad \text { in } E(\bar{s}) \backslash \bar{D} .
$$

Now we define, for $c>c_{*}$,

$$
\underline{u}(x)= \begin{cases}\max \left\{\omega_{\alpha(c)}(x), \underline{w}(x)\right\}, & x \in E(\hat{s}) \backslash D, \\ \omega_{\alpha(c)}(x), & x \in \mathbb{R}^{n} \backslash E(\hat{s}) .\end{cases}
$$

We know from (3.8) that

$$
\underline{u}=\underline{w}, \quad \text { in } E(\bar{s}) \backslash \bar{D},
$$

and in particular

$$
\underline{u}=\underline{w}=\varphi, \quad \text { on } \partial D .
$$

We know from (3.7) that $\underline{u}=\omega_{\alpha(c)}$ in a neighborhood of $\partial E(\hat{s})$. Therefore $\underline{u}$ is locally Lipschitz in $\mathbb{R}^{n} \backslash D$. Since both $\omega_{\alpha(c)}$ and $\underline{w}$ are viscosity subsolutions of (1.1) in $\mathbb{R}^{n} \backslash \bar{D}$, so is $\underline{u}$.

For $c>c_{*}$,

$$
\bar{u}(x):=\frac{1}{2} x^{T} A x+c
$$

is a smooth convex solution of (1.1). By (3.8),

$$
\omega_{\alpha(c)} \leq \beta \leq \widehat{b}<c_{*}<\bar{u}, \quad \text { on } \partial D .
$$

We also know by (3.6) that

$$
\lim _{|x| \rightarrow \infty}\left(\omega_{\alpha(c)}(x)-\bar{u}(x)\right)=0 .
$$


Thus, in view of the comparison principle for smooth $k$-convex solutions of (1.1) (see [4]), we have

$$
\omega_{\alpha(c)} \leq \bar{u}, \quad \text { on } \mathbb{R}^{n} \backslash D .
$$

By (3.7) and the above, we have, for $c>c_{*}$,

$$
w_{\xi} \leq \bar{u}, \quad \text { on } \partial(E(\hat{s}) \backslash D), \forall \xi \in \partial D .
$$

By the comparison principle for smooth convex solutions of (1.1), we have

$$
w_{\xi} \leq \bar{u}, \quad \text { in } E(\hat{s}) \backslash \bar{D}, \forall \xi \in \partial D .
$$

Thus

$$
\underline{w} \leq \bar{u}, \quad \text { in } E(\hat{s}) \backslash \bar{D} .
$$

This, combined with (3.11), implies that

$$
\underline{u} \leq \bar{u}, \quad \text { in } \mathbb{R}^{n} \backslash D .
$$

For any $c>c_{*}$, let $\mathcal{S}_{c}$ denote the set of $v \in \operatorname{USC}\left(\mathbb{R}^{n} \backslash D\right)$ which are viscosity subsolutions of (1.1) in $\mathbb{R}^{n} \backslash \bar{D}$ satisfying

$$
v=\varphi, \quad \text { on } \partial D,
$$

and

$$
\underline{u} \leq v \leq \bar{u}, \quad \text { in } \mathbb{R}^{n} \backslash D .
$$

We know that $\underline{u} \in \mathcal{S}_{c}$. Let

$$
u(x):=\sup \left\{v(x) \mid v \in \mathcal{S}_{c}\right\}, \quad x \in \mathbb{R}^{n} \backslash D .
$$

By (3.6) and the definitions of $\underline{u}$ and $\bar{u}$,

$$
u(x) \geq \underline{u}(x)=\omega_{\alpha(c)}(x)=\frac{1}{2} x^{T} A x+c+O\left(|x|^{\theta(2-n)}\right), \quad \text { as } \quad x \rightarrow \infty,
$$

and

$$
u(x) \leq \bar{u}(x)=\frac{1}{2} x^{T} A x+c .
$$

The estimate (1.4) follows.

Next, we prove that $u$ satisfies the boundary condition. It is obvious from (3.10) that

$$
\liminf _{x \rightarrow \xi} u(x) \geq \lim _{x \rightarrow \xi} \underline{u}(x)=\varphi(\xi), \quad \forall \xi \in \partial D .
$$

So we only need to prove that

$$
\limsup _{x \rightarrow \xi} u(x) \leq \varphi(\xi), \quad \forall \xi \in \partial D .
$$

Let $\omega_{c}^{+} \in C^{2}(\overline{E(\bar{s}) \backslash D})$ be defined by

$$
\begin{cases}\Delta \omega_{c}^{+}=0, & \text { in } E(\bar{s}) \backslash \bar{D} \\ \omega_{c}^{+}=\varphi, & \text { on } \partial D, \\ \omega_{c}^{+}=\max _{\partial E(\bar{s})} \bar{u}=\bar{s}+c, & \text { on } \partial E(\bar{s}) .\end{cases}
$$

It is easy to see that a viscosity subsolution $v$ of (1.1) satisfies $\Delta v \geq 0$ in the viscosity sense. Therefore, for every $v \in \mathcal{S}_{c}$, by $v \leq \omega_{c}^{+}$on $\partial(E(\bar{s}) \backslash D)$, we have

$$
v \leq \omega_{c}^{+}, \quad \text { in } E(\bar{s}) \backslash \bar{D} \text {. }
$$


It follows that

$$
u \leq \omega_{c}^{+}, \quad \text { in } E(\bar{s}) \backslash \bar{D}
$$

and then

$$
\limsup _{x \rightarrow \xi} u(x) \leq \lim _{x \rightarrow \xi} \omega_{c}^{+}(x)=\varphi(\xi), \quad \forall \xi \in \partial D .
$$

Finally, we prove that $u$ is a viscosity solution of (1.1). The following ingredients for the viscosity adaptation of Perron's method (see [14]) are available.

Lemma 3.2. Let $\Omega \subset \mathbb{R}^{n}$ be a bounded open set, $u \in \operatorname{LSC}(\bar{\Omega})$ and $v \in \operatorname{USC}(\bar{\Omega})$ are respectively viscosity supersolutions and subsolutions of (1.1) in $\Omega$ satisfying $u \geq v$ on $\partial \Omega$. Then $u \geq v$ in $\Omega$.

Under the assumptions $u, v \in C^{0}(\bar{\Omega})$, the lemma was proved in [25], based on Jensen approximations (see [15]). The proof remains valid under the weaker regularity assumptions on $u$ and $v$.

Lemma 3.3. Let $\Omega \subset \mathbb{R}^{n}$ be an open set, and let $\mathcal{S}$ be a nonempty family of viscosity subsolutions (supersolutions) of (1.1) in $\Omega$. Set

$$
u(x)=\sup (\inf )\{v(x) \mid v \in \mathcal{S}\}
$$

and let

$$
u^{*}\left(u_{*}\right)(x)=\lim _{r \rightarrow 0} \sup _{B_{r}}\left(\inf _{B_{r}}\right) u
$$

be the upper (lower) semicontinuous envelope of $u$. Then, if $u^{*}<\infty\left(u_{*}>-\infty\right)$ in $\Omega, u^{*}\left(u_{*}\right)$ is a viscosity subsolution (supersolution) of (1.1) in $\Omega$.

Lemma 3.3 can be proved by standard arguments; see e.g. [8]. With these ingredients, an application of the Perron process (see e.g. Lemma 4.4 in [8]) gives that $u \in C^{0}\left(\mathbb{R}^{n} \backslash D\right)$ is a viscosity solution of (1.3). Theorem 1.1 is established.

\section{ACKNOWLEDGEMENTS}

The first author was partially supported by NNSF (11071020) (11371060) and SRFDPHE (20100003110003). He also would like to thank the Department of Mathematics and the Center for Nonlinear Analysis at Rutgers University for their hospitality and stimulating environment. The second author was partially supported by SRFDPHE (20100003120005), NNSF (11071020) (11126038) and the Ky and Yu-Fen Fan Fund Travel Grant from the AMS. The work of the third author was partially supported by NSF grants DMS-0701545, DMS-1203961. They were all partially supported by the Fundament Research Funds for the Central Universities.

\section{REFERENCES}

[1] L. A. Caffarelli, Topics in PDEs: The Monge-Ampère equation. Graduate course, Courant Institute, New York University, 1995.

[2] Luis A. Caffarelli and Xavier Cabré, Fully nonlinear elliptic equations, American Mathematical Society Colloquium Publications, vol. 43, American Mathematical Society, Providence, RI, 1995. MR 1351007 (96h:35046) 
[3] L. Caffarelli and Yanyan Li, An extension to a theorem of Jörgens, Calabi, and Pogorelov, Comm. Pure Appl. Math. 56 (2003), no. 5, 549-583, DOI 10.1002/cpa.10067. MR.1953651 (2004c:35116)

[4] L. Caffarelli, L. Nirenberg, and J. Spruck, The Dirichlet problem for nonlinear second-order elliptic equations. III. Functions of the eigenvalues of the Hessian, Acta Math. 155 (1985), no. 3-4, 261-301, DOI 10.1007/BF02392544. MR806416 (87f:35098)

[5] Eugenio Calabi, Improper affine hyperspheres of convex type and a generalization of a theorem by K. Jörgens, Michigan Math. J. 5 (1958), 105-126. MR0106487 (21 \#5219)

[6] Shiu Yuen Cheng and Shing-Tung Yau, Complete affine hypersurfaces. I. The completeness of affine metrics, Comm. Pure Appl. Math. 39 (1986), no. 6, 839-866, DOI 10.1002/cpa.3160390606. MR 859275 (87k:53127)

[7] Kai-Seng Chou and Xu-Jia Wang, A variational theory of the Hessian equation, Comm. Pure Appl. Math. 54 (2001), no. 9, 1029-1064, DOI 10.1002/cpa.1016. MR1835381(2002e:35072)

[8] Michael G. Crandall, Hitoshi Ishii, and Pierre-Louis Lions, User's guide to viscosity solutions of second order partial differential equations, Bull. Amer. Math. Soc. (N.S.) 27 (1992), no. 1, 1-67, DOI 10.1090/S0273-0979-1992-00266-5. MR1118699 (92j:35050)

[9] Limei Dai, Existence of solutions with asymptotic behavior of exterior problems of Hessian equations, Proc. Amer. Math. Soc. 139 (2011), no. 8, 2853-2861, DOI 10.1090/S0002-99392011-10833-5. MR2801627 (2012d:35091)

[10] Limei Dai and Jiguang Bao, On uniqueness and existence of viscosity solutions to Hessian equations in exterior domains, Front. Math. China 6 (2011), no. 2, 221-230, DOI 10.1007/s11464-011-0109-x. MR2780888

[11] Philippe Delanoë, Partial decay on simple manifolds, Ann. Global Anal. Geom. 10 (1992), no. 1, 3-61, DOI 10.1007/BF00128337. MR.1172619 (93h:58144)

[12] L. Ferrer, A. Martínez, and F. Milán, An extension of a theorem by K. Jörgens and a maximum principle at infinity for parabolic affine spheres, Math. Z. 230 (1999), no. 3, 471-486, DOI 10.1007/PL00004700. MR.1679973 (2001d:53010)

[13] L. Ferrer, A. Martínez, and F. Milán, The space of parabolic affine spheres with fixed compact boundary, Monatsh. Math. 130 (2000), no. 1, 19-27, DOI 10.1007/s006050050084. MR.1762061 (2001e:53013)

[14] Hitoshi Ishii, On uniqueness and existence of viscosity solutions of fully nonlinear second-order elliptic PDEs, Comm. Pure Appl. Math. 42 (1989), no. 1, 15-45, DOI 10.1002/cpa.3160420103. MR 973743 (89m:35070)

[15] Robert Jensen, The maximum principle for viscosity solutions of fully nonlinear second order partial differential equations, Arch. Rational Mech. Anal. 101 (1988), no. 1, 1-27, DOI 10.1007/BF00281780. MR.920674 (89a:35038)

[16] Huaiyu Jian, Hessian equations with infinite Dirichlet boundary value, Indiana Univ. Math. J. 55 (2006), no. 3, 1045-1062, DOI 10.1512/iumj.2006.55.2728. MR2244597 (2008f:35120)

[17] Konrad Jörgens, Über die Lösungen der Differentialgleichung $r t-s^{2}=1$ (German), Math. Ann. 127 (1954), 130-134. MR0062326(15,961e)

[18] J. Jost and Y. L. Xin, Some aspects of the global geometry of entire space-like submanifolds, Results Math. 40 (2001), no. 1-4, 233-245. Dedicated to Shiing-Shen Chern on his 90th birthday. MR.1860371 (2002i:53070)

[19] Norman Meyers and James Serrin, The exterior Dirichlet problem for second order elliptic partial differential equations, J. Math. Mech. 9 (1960), 513-538. MR0117421 (22 \#8200)

[20] A. V. Pogorelov, On the improper convex affine hyperspheres, Geometriae Dedicata 1 (1972), no. 1, 33-46. MR.0319126(47 \#7672)

[21] Neil S. Trudinger, The Dirichlet problem for the prescribed curvature equations, Arch. Rational Mech. Anal. 111 (1990), no. 2, 153-179, DOI 10.1007/BF00375406. MR1057653 (91g:35118)

[22] Neil S. Trudinger, On the Dirichlet problem for Hessian equations, Acta Math. 175 (1995), no. 2, 151-164, DOI 10.1007/BF02393303. MR1368245 (96m:35113)

[23] Neil S. Trudinger, Weak solutions of Hessian equations, Comm. Partial Differential Equations 22 (1997), no. 7-8, 1251-1261, DOI 10.1080/03605309708821299. MR1466315 (99a:35077)

[24] Neil S. Trudinger and Xu-Jia Wang, The Bernstein problem for affine maximal hypersurfaces, Invent. Math. 140 (2000), no. 2, 399-422, DOI 10.1007/s002220000059. MR.1757001 (2001h:53016) 
[25] John I. E. Urbas, On the existence of nonclassical solutions for two classes of fully nonlinear elliptic equations, Indiana Univ. Math. J. 39 (1990), no. 2, 355-382, DOI 10.1512/iumj.1990.39.39020. MR.1089043 (92h:35074)

[26] C. Wang and J. G. Bao: Necessary and sufficient conditions on existence and convexity of solutions for Dirichlet problems of Hessian equations on exterior domains, Proc. Amer. Math. Soc. 141 (2013), no. 4, 1289-1296. MR3008876

School of Mathematical Sciences, Beijing Normal University, Laboratory of Mathematics and Complex Systems, Ministry of Education, Beijing 100875, People's Republic OF CHINA

E-mail address: jgbao@bnu.edu.cn

School of Mathematical Sciences, Beijing Normal University, Laboratory of Mathematics and Complex Systems, Ministry of Education, Beijing 100875, People's Republic OF CHINA

E-mail address: hgli@bnu.edu.cn

Department of Mathematics, Rutgers University, 110 Frelinghuysen Road, PiscatAWAY, NEW JERSEY 08854

E-mail address: yyli@math.rutgers.edu 